\title{
Evaluation of vertebral artery dominance, hypoplasia and variations in the origin: angiographic study in $\mathbf{2 5 4}$ patients
}

\author{
O. Ergun, I. Gunes Tatar, E. Birgi, B. Hekimoglu \\ Department of Radiology, Ankara Diskapi Yildirim Beyazit Training and Research Hospital, Ankara, Turkey \\ [Received: 1 April 2015; Accepted: 8 May 2015]
}

Background: The aim of this study was to determine the dimensional characteristics and variations in the origin of vertebral arteries (VA).

Materials and methods: We retrospectively reviewed angiographic studies in 254 patients (133 males, 121 females) for the evaluation of diameter differences in VA. We examined different criteria from the literature (difference of $\geq 0.3 \mathrm{~mm}$, $\geq 0.8 \mathrm{~mm}, \geq 1 \mathrm{~mm}$ between the widths of two VA and diameter ratio more than 1.4) to find out the dominant VA, rate of co-dominance and hypoplasia. The differences among groups were analysed using the $\chi^{2}$ and Kruskal-Wallis test. Also concordance analysis test was used to determine correspondence between the tests. We also noticed the variations in the origin of $V A$.

Results: The average diameter of VA in 254 patients was $3.21 \pm 0.7 \mathrm{~mm}$ on the right, and $3.16 \pm 0.7 \mathrm{~mm}$ on the left. The average diameter difference was found $0.88 \pm 0.7 \mathrm{~mm}$. The rate of hypoplasia was found $7.1 \%$ on the right and 9.4\% on the left. Among 254 patients according to the criterion of any diameter difference; right side was found wider in 126 (49.6\%) patients and left side was found wider in 120 (47.2\%) patients. The criterion of $0.3 \mathrm{~mm}$ or greater difference showed right VA dominance in 107 (42.1\%) patients, left VA dominance in 99 (39\%) patients. Co-dominance was mainly observed when we used the criteria of $0.8 \mathrm{~mm}$ and $1 \mathrm{~mm}$ or greater difference and diameter ratio more than 1.4. We found out harmony of two criterion of difference of $\geq 0.8 \mathrm{~mm}$ and $\geq 1 \mathrm{~mm}$ (concordance analysis test, 76.1\%). There was no statistically significant relation between age, gender and any dominance criteria $(p>0.05)$. The majority of VA showed classical origin arising from both subclavian arteries with a rate of $94.9 \%$. Conclusions: The most striking result we have found is the dominance of the right VA in diameter by using all different criteria unlike with previous reports in the literature. (Folia Morphol 2016; 75, 1: 33-37)

Key words: anatomic variation, angiography, vertebral artery

\section{INTRODUCTION}

The role of vertebral arteries (VA) in brain's blood supply is a well-known entity. Any change in VA haemodynamics may cause important disorders in cerebellum, brain stem, inner ear and spinal cord $[6,9]$.
VA are mainly being examined by colour Doppler ultrasonography and some important parameters such as lumen diameter, mean blood flow velocities, flow side and blood flow volume are obtainable [18]. VA can be measured at the V2 segment, between

Address for correspondence: O. Ergun, MD, Dışkapı Yıldırım Beyazıt Eğitim ve Araştırma Hastanesi, Dışkapı, Ankara, Turkey, tel: 0090312 5962618, fax: 0090312 3186690, e-mail: onurergun@yahoo.com 
5th and 6th cervical vertebrae. Demonstration of the origin of VA with Doppler ultrasonography is difficult in majority of the patients. Digital subtraction angiography (DSA) is the gold standard modality in visualisation of the entire segment of VA. Ischaemic stroke of posterior territory circulation is a result of atherosclerosis and congenital disorders [9]. Also symptoms like vertigo, migraine and tinnitus are found to be related with VA diameter in some studies [15]. For these reasons during cerebral angiography besides the carotid angiogram it is essential to perform vertebral angiography in detection of ischaemic events. Diameter differences, stenotic or occlusive segments and variations in the origin of the VA are clearly shown with DSA.

The endovascular approach for supra-aortic lesions has been successful and is now a widely accepted alternative to surgery. Especially during stenting of subclavian arteries and aortic arch it might be important to know the dominant VA in decision of which one of the vertebral or subclavian arteries could be sacrificed in case of proximity to the lesion [5].

The aim of this study was to determine the dimensional characteristics and variations in the origin of VA. We examined different criteria from the literature to find out the side of dominant $\mathrm{VA}$, rate of co-dominance and hypoplasia.

\section{MATERIALS AND METHODS}

We retrospectively reviewed angiographic studies in 254 patients from January 2012 to December 2013, for the evaluation of diameter differences in VA. We also noticed the variations in the origin of VA. We excluded the patients with subclavian-carotid steal syndromes and occluded VA. Angiographies belonged to $133(52.4 \%)$ male and $121(47.6 \%)$ female patients. The mean age of the patients was 59.5 (range: 13-88) years. The mean age was 58.9 years in female patients (range: 13-86 years) and 60 years in male patients (range: $14-88$ years). Vertebral artery diameter was measured by two different radiologists at different times, and average values were taken. Aortic arch angiograms which were taken in 30 degrees left anterior oblique position were used for the measurements and measurements were made on V1 segment of VA. Hypoplasia and dominance of VA in males and females were classified according to the criteria in the literature and the most common origin variations were also demonstrated. After a review of the literature based on VA studies, we have found different diameter criteria to establish the dominant VA. Studies using difference of any diameter, difference of $\geq 0.3 \mathrm{~mm}$, difference of $\geq 0.8 \mathrm{~mm}$, difference of $\geq 1 \mathrm{~mm}$ between the widths of two VA and also diameter ratio more than 1.4 were determined. We have achieved our results according to all these criteria. We decided hypoplasia criterion as $\leq 2 \mathrm{~mm}$ like majority of the studies in the literature.

Data were analysed descriptively, and the differences among groups were analysed using the $\chi^{2}$ and Kruskal-Wallis test. Also concordance analysis test was used to determine correspondence between the tests. Statistical significance was considered as $p<0.05$.

\section{RESULTS}

The average diameter of VA in 254 patients was $3.22 \pm 0.7 \mathrm{~mm}$ on the right, and $3.16 \pm 0.8 \mathrm{~mm}$ on the left. The average diameter difference was found $0.89 \pm 0.7 \mathrm{~mm}$. The average diameter of VA in female patients was $3.15 \pm 0.7 \mathrm{~mm}$ on the right, and $3.18 \pm$ $\pm 0.8 \mathrm{~mm}$ on the left. The average diameter of VA in male patients was $3.27 \pm 0.7 \mathrm{~mm}$ on the right, and $3.14 \pm 0.8 \mathrm{~mm}$ on the left.

The rate of hypoplasia was found $7.1 \%(18 / 254)$ on the right and $9.4 \%$ (24/254) on the left. In females, the rate of hypoplasia was found $8.3 \%$ on the right and $9.1 \%$ on the left. In males, the rate of hypoplasia was found $6 \%$ on the right and $9.8 \%$ on the left.

Among 254 patients, according to the criterion of any diameter difference, right side was found wider in $126(49.7 \%)$ patients and left side was found wider in $120(47.2 \%)$ patients. VA were equal in diameter in $8(3.1 \%)$ patients.

A difference of $0.3 \mathrm{~mm}$ or greater between the width of 2 VA showed right VA dominance in 107 $(42.1 \%)$ patients, left VA dominance in $99(39 \%)$ patients. 48 (18.9\%) patients were found to be equal.

Co-dominance takes the first place when we use the criterion of $0.8 \mathrm{~mm}$ or greater difference between the width of 2 VA, with the number of 129 (50.8\%) patients. Right VA was found to be dominant in $67(26.4 \%)$ patients and left VA was found to be dominant in $58(22.8 \%)$ patients.

The criterion of $1 \mathrm{~mm}$ or greater difference showed similar results in ranking with different ratios. Co-dominance was found in majority of the patients with a number of $159(62.6 \%)$ patients. Right VA was found to be dominant in $51(20.1 \%)$ patients and left VA was found to be dominant in $44(17.3 \%)$ patients. 
Table 1. Different diameter criteria in the literature to establish the dominant vertebral artery (VA) identified by digital subtraction angiography among 254 patients: all criteria are summarised with number of patients and ratio of each side

\begin{tabular}{lcccccc}
\hline Criterion & \multicolumn{2}{c}{ Co-dominance } & \multicolumn{2}{c}{ Right VA } & \multicolumn{2}{c}{ Left VA } \\
\hline Difference in any diameter & 8 & $3.1 \%$ & 126 & $49.7 \%$ & 120 & $47.2 \%$ \\
Difference of $\geq 0.3 \mathrm{~mm}$ & 48 & $18.9 \%$ & 107 & $42.1 \%$ & 99 & $39 \%$ \\
Difference of $\geq 0.8 \mathrm{~mm}$ & 129 & $50.8 \%$ & 67 & $26.4 \%$ & 58 & $22.8 \%$ \\
Difference of $\geq 1 \mathrm{~mm}$ & 159 & $62.6 \%$ & 51 & $20.1 \%$ & 44 & $17.3 \%$ \\
Diameter ratio more than 1.4 & 168 & $66.1 \%$ & 45 & $17.8 \%$ & 41 & $16.1 \%$ \\
\hline
\end{tabular}

The results of diameter ratio (ipsilateral/contralateral > 1.4) were alike with the last two criteria. Co-dominance in 168 (66.1\%) patients, right dominance in $45(17.8 \%)$ patients and left dominance in $41(16.1 \%)$ patients were found.

All criteria are summarised with number of patients and ratio of each side in Table 1.

There was no statistically significant relation between age, gender and any dominance criteria ( $p>0.05)$.

The majority of VA showed classical origin arising from both subclavian arteries with a rate of $94.9 \%$ (241/254). Left VA with a direct origin from the aortic arch was found in 10 (3.9\%) patients (7 males and 3 females) and right VA arising from aberrant right subclavian artery was found in $3(1.2 \%)$ patients (2 females and 1 male).

\section{DISCUSSION}

Vertebral arteries in the brain's blood supply has a significant place as being the second largest blood supplier. In case of failure of the carotid artery system with occlusive diseases, VA provide the circulation by collateral pathways [9].

In the literature vertebral arteries are investigated by colour Doppler ultrasound, anatomic studies, computed tomography angiography (CTA) and conventional angiography in many years [10, 11, 14-16]. B-mode and colour Doppler ultrasound is the first-step method in evaluation of vertebrobasilar system. Parameters such as lumen diameter, mean blood flow velocities, flow side and blood flow volume can be measured easily and non-invasively [18]. Except these values, the origin of the VA is important whether it is stenotic or occluded, and also variations may occur. It is difficult to demonstrate the origin of VA by ultrasound in most cases. Morphologic and haemodynamic characteristics of the VA are important; because neurologic symptoms are associated with common pathological conditions of VA in many patients [15].
Diameter differences in right and left VA had been studied and dominant side was explained by different criteria in the literature. Zwiebel [18] found that left VA is wider than right VA in $73 \%$ of a healthy population and announced this as left VA dominance. Turan-Ozdemir et al. [15] showed similar results of left VA dominance in 64\% (55/85 patients) with colour Doppler ultrasound. There are also studies reporting no differences in diameter of VA or indicating right VA is wider than left [13]. An anatomic study of intracranial parts of VA in a series of 11 cadavers by Akar et al. [1] showed right VA dominance with a rate of $45.4 \%$. The results in studies mentioned above are obtained by the criterion of any diameter difference between VA. In our study when we use this criterion, right side was found wider in $49.7 \%$ of the patients and left side was found wider in $47.2 \%$ of the patients.

Another criterion in selection of the dominant side was used by Hong et al. [4] in a study about basilar artery curvature and peri-vertebrobasilar junctional infarcts. In this study dominant VA was defined with criterion of side to side diameter difference $\geq 0.3 \mathrm{~mm}$ in CTA and left VA was found dominant with a rate of $69.2 \%(63 / 91)$. But in our study, using this criterion, right VA was dominant in $42.1 \%$ of the patients and left VA was dominant in $39 \%$ of the patients.

Grasso et al. [3] observed left VA at least $0.8 \mathrm{~mm}$ wider than right VA in 4 of 6 brains in an anatomical study. In our study, when we use this criterion, right VA was found to be dominant in $26.4 \%$ of the patients and left VA was found to be dominant in $22.8 \%$ of the patients.

Songur et al. [14] measured the widths of the VA in a large series of an anatomic study with 109 autopsies and difference of $1 \mathrm{~mm}$ or greater between the widths of VA was accepted as the dominancy criterion. Left dominancy was observed in $21.2 \%$ and right dominancy was observed in $17.3 \%$ [14]. With this criterion, in our study right VA was found to be 
dominant in $20.1 \%$ of the patients and left VA was found to be dominant in $17.3 \%$ of the patients. Also in the same study, VA width was evaluated as right hypoplasia in $20.2 \%$ and left hypoplasia in $14.4 \%$ with the criterion of $<2 \mathrm{~mm}$ diameter of VA [14]. Our result of hypoplasia rates is rather different from the study above. We found right VA hypoplasia in $7.1 \%$ and left VA hypoplasia in $9.4 \%$ of the patients.

In a study of Yokoyama et al. [17] the dominant VA was defined with the diameter ratio of ipsilateral and contralateral VA more than 1.4; to classify the dissecting VA whether in dominant or non. When we use this criterion to evaluate dominant side, we achieved the right VA with a rate of $17.8 \%$ and left VA with a rate of $16.1 \%$.

Considering the all criteria; the most striking result we found is the dominance of the right VA in diameter unlike with numerous previous reports in the literature mentioned above.

In the literature there are different criteria for the diagnosis of the dominant vertebral artery as we mentioned above. When we use the concordance analysis test; we have found out harmony of two criterion of difference of $\geq 0.8 \mathrm{~mm}$ and difference of $\geq 1 \mathrm{~mm}$ between the widths of 2 VA (76.1\%). But we couldn't find the gold standard criterion statistically with just only diameter. We think that diameter could give an idea in evaluation of the dominance but it must be also supported by velocities and blood flow volume.

Without any conclusive evidence, variations in the origin of the VA are thought to change haemodynamics and expose to intracranial aneurysms. Satti et al. [12] stated that; patients should be screened to bring out potential presence of intracranial aneurysms due to variations in origin of VA. As shown in cadavers that direct origin of left VA from aortic arch (type 3 aortic arch) tends to increase blood flow in left VA and incidence of cerebrovascular diseases [11]. Komiyana et al. [7] reported higher rates of VA dissection in cases of left VA originating from the aortic arch.

Uchino et al. [16] identified the variations in VA origin with CTA among 2287 patients. Direct aortic origin of the left VA between the left common carotid artery and left subclavian artery was found $4.1 \%$ which is the most seen variation and called as type 3 aortic arch in most of the studies. At the same study right VA originating from aberrant right subclavian artery prevalence was $0.4 \%$ [16]. In our study there were 13 patients with variations in the origin of the VA (5.1\%). Left VA with a direct origin from the aortic arch in 10 (3.9\%) patients and right VA arising from aberrant right subclavian artery in $3(1.2 \%)$ patients were detected. There was no direct aortic origin of the right VA in our study.

Celikyay et al. [2] also defined two different variations of the left $\mathrm{VA}$ depending on the direct origin from the aortic arch before or after the left subclavian artery. In this CTA based study with 1136 patients; left VA arising from the aortic arch before left subclavian artery has been reported in $33(2.9 \%)$ patients, and left VA arising from the aortic arch after left subclavian artery has been reported in only $1(0.1 \%)$ patient [2]. Similarly in the literature; left VA arising from the aortic arch before the left subclavian artery has been observed with a higher rate [8]. Also in our study all left VA with a direct origin from the aortic arch were arising before the left subclavian artery.

Our study has some limitations. It is a retrospective study performed with only the data of VA diameter and without any flow parameters. Also the number of the patients might be insufficient for such a study about anatomical variations.

\section{CONCLUSIONS}

In conclusion, to be aware of which VA is dominant is very important especially in the interventions in which one of the subclavian arteries must be sacrificed. There are various diameter criteria in the literature for the determination of VA dominance. In our study, interestingly, we found that in most cases right VA was dominant in all the groups formed by using different criteria. When we use the criteria of $\geq 0.8 \mathrm{~mm}$ and $\geq 1 \mathrm{~mm}$ difference between the widths of $2 \mathrm{VA}$, diameter ratio of ipsilateral/contralateral $>1.4$, co-dominance was found in the majority of patients, but the dominant side didn't change. Our investigation showed dominance of the right VA in diameter unlike with numerous previous reports in the literature.

Radiologists should also be familiar to the variations in the origin of VA to prevent complications before surgery and catheterisation failure during angiographic studies.

\section{REFERENCES}

1. Akar ZC, Dujovny M, Slavin KV (1994) Microsurgical anatomy of the intracranial part of the vertebral artery. Neurol Res, 16: 171-180.

2. Celikyay ZR, Koner AE, Celikyay F, Deniz C, Acu B, Firat MM (2013) Frequency and imaging findings of variations in human aortic arch anatomy based on multidetector computed tomography data. Clin Imag, 37: 1011-1019. 
3. Grasso G, Alafaci C, Passalacqua M, Tschabitscher M, Salpietro FM, Tomasello F (2005) Landmarks for vertebral artery repositioning in bulbar compression syndrome: anatomic and microsurgical nuances. Neurosurgery, 56: 160-164.

4. Hong JM, Chung CS, Bang OY, Yong SW, Joo IS, Huh K (2009) Vertebral artery dominance contributes to basilar artery curvature and peri-vertebrobasilar junctional infarcts. J Neurol Neurosurg Psychiatry, 80: 1087-1092.

5. Jacobs D, Lajos P (2008) Endovascular technique for percutaneous stent graft repair of subclavian artery pseudoaneurysm. Available via CTSNet. http://www.ctsnet.org/ sections/clinicalresources/clinicalcases/article- 21 .

6. Kim JS, Lee H (2009) Inner ear dysfunction due to vertebrobasilar ischemic stroke. Semin Neurol, 29: 534-540.

7. Komiyana M, Morikawa T, Nakjima H, Nishikawa M, Yasui T (2001) High incidence of dissection associated with left vertebral artery of aortic origin. Neurol Med Chir, 41: 8-11.

8. McDonald JJ, Anson BJ (1940) Variations in the origin of arteries derived from the aortic arch, in American white and negroes. Am J Phys Anthropol, 27: 91-107.

9. Morovic S, Skaric-Juric T, Demarin V (2007) Morphologic and hemodynamic characteristics of vertebral arteries in men and women. Rad Za Medicinske Znanosti, 121-127.

10. Müller M, Schmitz BL, Pauls S, Schick M, Röhrer S, Kapapa T, Schlötzer W (2011) Variations of the aortic arch: a study on the most common branching patterns. Acta Radiol, 52: 738-742.
11. Patil ST, Meshram MM, Kamdi NY, Kasote AP, Parchand MP (2012) Study on branching pattern of aortic arch in Indian. Anat Cell Biol, 45: 203-206.

12. Satti SR, Cerniglia CA, Koenigsberg RA (2007) Cervical Vertebral artery variations: an anatomic study. Am J Neuroradiol, 28: 976-980.

13. Scheel P, Ruge C, Schoning M (2000) Flow velocity and flow volume measurements in the extracranial carotid and vertebral arteries in healthy adults: reference data and the effects of age. Ultrasound Med Biol, 26: 1261-1266.

14. Songur A, Gonul Y, Ozen OA, Kucuker H, Uzun I, Bas O, Toktas M (2008) Variations in the intracranial vertebrobasilar system. Surg Radiol Anat, 30: 257-264.

15. Turan-Ozdemir S, Yıldız C, Cankur NS (2002) Evaluation of vertebral artery system in a healthy population by using colour duplex Doppler ultrasonography. (in Turkish). Uludag Univ Tıp Fak Derg, 28: 95-99.

16. Uchino A, Saito N, Takahashi M, Okada Y, Kozawa E, Nishi N, Mizukoshi W, Nakajima R, Watanabe Y (2013) Variations in the origin of the vertebral artery and its level of entry into the transverse foramen diagnosed by $\mathrm{CT}$ angiography. Neuroradiology, 55: 585-594.

17. Yokoyama $Y$, Fujimoto $S$, Toyoda $K$, Inoue $T$, Jinnouchi J, Nakachi k, Gotoh S, Fukui D, Yasumori K, Okada Y (2005) Relationships between vertebral artery diameter and dissecting aneurysm formation. J Cerebral Blood Flow Metab, 25: 148.

18. Zwiebel WJ (1986) Introduction to vascular ultrasonography. 2nd Ed. W.B. Saunders, Philadelphia. 